\title{
Nonlocal type asymptotic behavior for solutions of second order difference equations
}

\section{Cristóbal González and Antonio Jiménez-Melado* (1)}

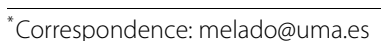
Dept. Análisis Matemático, Estadística e I.O., y Matemática Aplicada, Fac. Ciencias, Univ. Málaga, Málaga, 29071, Spain

\section{Abstract}

$$
\left\{\begin{array}{l}
\Delta(q \Delta \mathbf{x})(n)+f(n+1, \mathbf{x}(n+1))=0, \\
\mathbf{x}(\infty)=g(\mathbf{x}),
\end{array}\right.
$$

in the context of an arbitrary Banach space $\left(X,\|\cdot\|_{X}\right)$, and we give sufficient conditions that ensure the existence of solutions to it.

In order to present our result, we shall need to study conditions that ensure the existence of solutions with a nonlocal asymptotic behavior for the following equation:

$$
\mathbf{x}(n)=g(\mathbf{x})-\sum_{k=n+1}^{\infty} \sum_{j=n}^{k-1} \frac{1}{q(j)} f(k, \mathbf{x}(k)) .
$$

MSC: 39A22; 39B99; 47N20

Keywords: nonlocal problem; asymptotic behavior; second order difference equation; Leray-Schauder type fixed point theorem

\section{Introduction}

Throughout this paper $\mathbb{N}, \mathbb{R}$, and $\mathbb{R}_{+}$will denote the usual sets of numbers, i.e., nonnegative integers, reals, and nonnegative reals, respectively. Also, $\left(X,\|\cdot\|_{X}\right)$ will denote an arbitrary (real) Banach space, whose (open) balls (with center $x \in X$ and radius $r$ ) are $B(x, r)$, or $B_{X}(x, r)$ depending on whether the base space needs emphasis or not. The closure, the convex hull, and the closed convex hull of a given set $A \subset X$ are written $\bar{A}, \operatorname{co}(A)$, and $\overline{c o}(A)$, respectively. Sequences (or double sequences) will be understood as mappings defined on $\mathbb{N}($ or $\mathbb{N} \times \mathbb{N}$ ), thus avoiding as much as possible the use of subscripts or superscripts. For instance, a sequence in $X$ will be a mapping $\mathbf{x}: \mathbb{N} \rightarrow X$, whose $n$th component is $\mathbf{x}(n)$. The operator forward difference, $\Delta$, applies sequences into sequences. Thus, if $\mathbf{x}$ is a sequence, then $\Delta \mathbf{x}$ is a sequence that component-wise is given by $\Delta \mathbf{x}(n)=\mathbf{x}(n+1)-\mathbf{x}(n)$. Rather than considering the whole space of sequences on $X$, we shall be working with $\ell_{\infty}(X)$, the usual 
space of bounded sequences on $X$, which becomes a Banach space under the sup norm: $\|\mathbf{x}\|_{\infty}=\sup _{n}\|\mathbf{x}(n)\|_{X}$.

The study of difference equations in the context of Banach spaces has always attracted the attention of several authors (see for instance [1] and the references therein). In this paper, we follow this line of work.

For $q$ a given sequence of positive real numbers and $f$ an $X$-valued function defined on $\mathbb{N} \times X$, we look, in this paper, for conditions that ensure the existence of bounded $X$-valued sequences $\mathbf{x}$, solutions of the following problem:

$$
\left\{\begin{array}{l}
\Delta(q \Delta \mathbf{x})(n)+f(n+1, \mathbf{x}(n+1))=0, \\
\mathbf{x}(\infty)=g(\mathbf{x}) .
\end{array}\right.
$$

Here, $\mathbf{x}(\infty)=\lim _{n \rightarrow \infty} \mathbf{x}(n)$ and $g$ is an $X$-valued function defined on $\ell_{\infty}(X)$. This nonlocal asymptotic behavior, as opposed to local asymptotic behavior given by $\mathbf{x}(\infty)=$ constant, is the interesting part of our study and makes it different from what, we believe, has been done before. For instance, in [2], a quite general set of hypotheses was given in order to ensure the existence of solutions with a specified local asymptotic behavior for the above equation (see also [3-9]). Those hypotheses were enough in order to use the Sadovskii fixed point theorem, but when we tried to adapt them to the nonlocal setting, we did not see how to do it without imposing conditions difficult to verify. In the end, we have realized that a Leray-Schauder type fixed point theorem is the appropriate tool to obtain a good result for this nonlocal setting. The basics of this theory are briefly explained in Section 2, together with a result on fixed points of mappings defined on sequence spaces which is similar to Theorem 1 in [2].

In Section 3, we shall study the following summation equation:

$$
\mathbf{x}(n)=g(\mathbf{x})-\sum_{k=n+1}^{\infty} \sum_{j=n}^{k-1} \frac{1}{q(j)} f(k, \mathbf{x}(k)),
$$

whose solutions, as one can easily check, are also solutions of the problem (1), because the convergence of the series is implicitly assumed in this direction. In the other direction, one needs to provide conditions ensuring the convergence of the series in order to see that the solutions to problem (1) are also solutions of the above summation equation. Actually, an equation a bit more general than (2) will be the main object of our study. The paper will be complete once we present the corresponding result to obtain solutions of problem (1).

\section{A fixed point theorem}

Compactness is a usual ingredient when trying to obtain results about fixed points of mappings. Without going any further, the well-known Schauder fixed point theorem asserts that a compact mapping, i.e., a continuous mapping that transforms bounded sets into relatively compact ones, has a fixed point, whenever it leaves invariant a nonempty, bounded, closed, and convex set. The invariance of the set can be relaxed if we refer to an also wellknown Leray-Schauder type fixed point theorem, which states that a compact mapping $T$ has a fixed point if it satisfies the Leray-Schauder boundary condition on $\bar{B}(0, R)$, for some $R>0$, that is, if there exists $R>0$ such that

$$
T(x) \neq \lambda x, \quad \text { whenever }\|x\|=R \text {, and } \lambda>1 .
$$


A natural generalization of compactness in these results deal with the concept of measures of noncompactness. A typical one is the measure of noncompactness of Kuratowski, defined for each bounded set $A$ as the infimum $\alpha(A)$ of the $\varepsilon>0$ for which $A$ admits a finite $\varepsilon$-cover. (By an $\varepsilon$-cover of $A$ we mean a collection of sets $F_{i}, i \in \mathcal{I}$, of diameter less than $\varepsilon$, whose union contains $A$.)

$$
\alpha(A)=\inf \left\{\varepsilon>0: A \subseteq \bigcup_{i=1}^{n} F_{i}, \operatorname{diam}\left(F_{i}\right)<\varepsilon\right\} .
$$

In general, a measure of noncompactness in $X$ is a mapping $m$ that assigns to each bounded set in $X$ a nonnegative real number, such that

(MNC1) for all bounded $A \subset X, m(A)=0$ if and only if $\bar{A}$ is compact;

(MNC2) for all bounded $A \subset X, m(A)=m(\bar{A})$;

(MNC3) for all bounded $A, B \subset X, m(A \cup B)=\max \{m(A), m(B)\}$.

A property deduced easily from (MNC3) is that if $A \subseteq B$ are bounded, then $m(A) \leq$ $\max \{m(A), m(B)\}=m(A \cup B)=m(B)$. Additionally, the measure of noncompactness of Kuratowski also satisfies the following useful properties:

(K1) for all bounded $A \subset X$ and all scalars $\lambda, \alpha(\lambda A)=|\lambda| \alpha(A)$;

(K2) for all bounded $A, B \subset X, \alpha(A+B) \leq \alpha(A)+\alpha(B)$;

(K3) for all bounded $A \subset X, \alpha(\operatorname{co}(A))=\alpha(A)$.

Compact mappings are particular cases of $m$-condensing mappings. These are continuous mappings $T$ that send bounded sets $A$ with $m(A)>0$ into bounded sets $T(A)$ with $m(T(A))<m(A)$. In this respect, in 1967, Sadovskii [10] obtained an extension of Schauder's fixed point theorem for condensing mappings, namely, if the measure of noncompactness is such that $m(A)=m(\operatorname{co}(A))$ for any bounded set $A \subset X$, then any condensing mapping has a fixed point, whenever it leaves invariant a nonempty, bounded, closed, and convex set. Again, the invariance of the set can be relaxed via a Leray-Schauder type fixed point theorem: if the measure of noncompactness is unaltered after applying convex hulls, we see that any condensing mapping has a fixed point if it satisfies the LeraySchauder boundary condition (LS) on $\bar{B}_{X}(0, R)$, for some $R>0$. For proofs of these results, at least for the Kuratowski measure of noncompactness, see, for instance, [11] and [12].

From now on, we restrict ourselves to the Kuratowski measures of noncompactness $\alpha_{X}$ in $X$, and $\alpha_{\infty}$ in $\ell_{\infty}(X)$, respectively. In 2003, the authors [2] used Sadovskii fixed point theorem in order to obtain a result as regards fixed points for mappings on sequence spaces.

Theorem A Let $\mathbf{K} \subset \ell_{\infty}(X)$ be a nonempty, bounded, closed, and convex set. Assume that a mapping $\mathbf{T}: \mathbf{K} \rightarrow \mathbf{K}$ is given by $\mathbf{T}=\left(T_{0}, T_{1}, \ldots\right)$, where each $T_{n}$ is $\left(\alpha_{\infty}, \alpha_{X}\right)$-condensing, and $\lim _{n} \operatorname{diam}\left(T_{n} \mathbf{K}\right)=0$. Then $\mathbf{T}$ has a fixed point in $\mathbf{K}$.

The proof just consisted in showing that $\mathbf{T}$ is $\alpha_{\infty}$-condensing. Now, we shall need a result which is a Leray-Schauder counterpart of Theorem A, in order to relax the existence of a $\mathbf{T}$-invariant set. This observation is just stated as our first result, for which there is no need to repeat the proof.

Theorem 1 Let $\mathbf{T}: \ell_{\infty}(X) \rightarrow \ell_{\infty}(X), \mathbf{T}=\left(T_{0}, T_{1}, \ldots\right)$, be such that maps bounded sets into bounded sets. Assume that each $T_{n}$ is $\left(\alpha_{\infty}, \alpha_{X}\right)$-condensing, and that $\lim _{n} \operatorname{diam} T_{n}(\mathcal{U})=0$ for each bounded set $\mathcal{U} \in \ell_{\infty}(X)$. Then $\mathbf{T}$ is $\alpha_{\infty}$-condensing. 
If, besides, $\mathbf{T}$ satisfies the Leray-Schauder boundary condition (LS) on $\bar{B}_{\ell_{\infty}(X)}(\mathbf{0}, R)$, for some $R>0$, then $\mathbf{T}$ has a fixed point.

\section{Solutions for a summation equation}

As we said in the Introduction, we will study an equation a bit more general than (2), in the context of the Banach space $X$,

$$
\mathbf{x}(n)=g(\mathbf{x})-\sum_{k=n+1}^{\infty} P(n, k) f(k, \mathbf{x}(k))
$$

where $g: \ell_{\infty}(X) \rightarrow X, P: \mathbb{N} \times \mathbb{N} \rightarrow \mathbb{R}_{+}$, and $f: \mathbb{N} \times X \rightarrow X$.

Observe that (2) is just the case $P(n, k)=\sum_{j=n}^{k-1} \frac{1}{q(j)}$, for $k \geq n+1$.

In order to ensure the existence of solutions to this equation we have found that the following conditions fit our requirements and are quite general:

(H1) $g: \ell_{\infty}(X) \rightarrow X$ is compact and bounded.

(H2) There exists a sequence $p: \mathbb{N} \rightarrow \mathbb{R}_{+}$such that

$$
P(n, k) \leq p(k), \quad \text { for all } n \in \mathbb{N} \text { and all } k \geq n+1 .
$$

(H3) There exist $\varphi: \mathbb{R}_{+} \rightarrow \mathbb{R}_{+}$, nondecreasing, and $a: \mathbb{N} \rightarrow \mathbb{R}_{+}$such that

$$
\|f(n, x)\|_{X} \leq a(n) \varphi\left(\|x\|_{X}\right), \quad \text { for all } n \in \mathbb{N} \text { and all } x \in X
$$

(This implies that each $f(n, \cdot)$ maps bounded sets into bounded sets.)

(H4) $\varphi(s)>0$ for all $s>0$, and $\int_{1}^{\infty} \frac{1}{\varphi(s)} d s=+\infty$.

(H5) $\sum_{n=0}^{\infty} p(n) a(n)<\infty$.

(H6) For $k \in \mathbb{N}, f(k, \cdot)$ is continuous on $X$, there exists $L_{k} \geq 0$ such that

$$
\alpha_{X}(f(k, A)) \leq L_{k} \alpha_{X}(A), \quad \text { for all bounded } A \subset X \text {, and } \sum_{k=0}^{\infty} p(k) L_{k}<1
$$

Observe that if each $f(k, \cdot)$ were a compact function then we could take $L_{k}=0$ for all $k$ in (H6). Also, notice that (H3) and (H5) tell us that $\sum_{k} p(k) f(k, \mathbf{x}(k))$ converges absolutely for each $\mathbf{x} \in \ell_{\infty}(X)$, and it does it uniformly on each bounded set of $\ell_{\infty}(X)$. Precisely, this convergence condition will help us to show that any solution of (1) is also a solution of (2). Recall that we mentioned before that the other direction was straightforward.

Proposition 2 Let $\mathbf{x} \in \ell_{\infty}(X)$ be a solution of (1). Put $P(n, k)=\sum_{j=n}^{k-1} \frac{1}{q(j)}($ for $k \geq n+1)$, and $p(k)=\sum_{j=0}^{k-1} \frac{1}{q(j)}($ for $k \geq 1)$. Assume that conditions (H3) and (H5) hold, as well as $\lim _{k} p(k)=\infty$. Then $\mathbf{x}$ is also a solution of (2).

Remark 1 The hypothesis $\sum_{j} \frac{1}{q(j)}=\infty(p(k) \rightarrow \infty)$ becomes natural if we think of $q(j)$ as equal to 1 for all $j$. Equation (1) would then be

$$
\Delta^{2} \mathbf{x}(n)=-f(n+1, \mathbf{x}(n+1)) .
$$


Proof For fixed $n$ and $N \geq n$ we have

$$
q(N+1) \Delta \mathbf{x}(N+1)-q(n) \Delta \mathbf{x}(n)=\sum_{k=n}^{N} \Delta(q \Delta \mathbf{x})(k)=-\sum_{k=n+1}^{N+1} f(k, \mathbf{x}(k)) .
$$

Recall that $q(n)>0$. So we may divide the above expression by $q(n)$, and since $\frac{1}{q(n)} \leq p(k)$ for $k \geq n+1$, the series in the resulting right hand side converges absolutely, by (H3) and (H5). So, taking limits as $N \rightarrow \infty$, we conclude that there exists the limit $y \in X$ of $q(N) \Delta \mathbf{x}(N)$ as $N \rightarrow \infty$.

We claim that this limit must be $y=0$. For, assuming $y \neq 0$, by Hahn-Banach, a continuous (real) linear functional $H$ exists on $X$ such that $H(y)=1$. This shows that $q(N) H(\Delta \mathbf{x}(N)) \rightarrow H(y)=1$, and hence, there exists $N_{0} \in \mathbb{N}$ such that $H(\Delta \mathbf{x}(N))>\frac{1}{2 q(N)}$ for all $N \geq N_{0}$. Thus, for $N \geq N_{0}$,

$$
H(\mathbf{x}(N+1))=H\left(\mathbf{x}\left(N_{0}\right)\right)+\sum_{j=N_{0}}^{N} H(\Delta \mathbf{x}(j))>H\left(\mathbf{x}\left(N_{0}\right)\right)+\frac{1}{2} \sum_{j=N_{0}}^{N} \frac{1}{q(j)},
$$

leaving us with a contradiction at the moment of taking limits as $N \rightarrow \infty$, for the left hand side converges to $H(\mathbf{x}(\infty))=H(g(\mathbf{x}))$ while the right hand side goes to $\infty$ because, by hypothesis, $\sum_{j} \frac{1}{q(j)}=\infty$.

Once the claim is proved, (3) yields

$$
\Delta \mathbf{x}(n)=\frac{1}{q(n)} \sum_{k=n+1}^{\infty} f(k, \mathbf{x}(k)), \quad \text { for all } n \in \mathbb{N}
$$

and again, for fixed $n$ and $N \geq n$,

$$
\begin{aligned}
\mathbf{x}(N+1)-\mathbf{x}(n) & =\sum_{j=n}^{N} \Delta \mathbf{x}(j)=\sum_{j=n}^{N} \frac{1}{q(j)} \sum_{k=j+1}^{\infty} f(k, \mathbf{x}(k)) \\
& =\sum_{k=n+1}^{N+1} \sum_{j=n}^{k-1} \frac{1}{q(j)} f(k, \mathbf{x}(k))+\sum_{k=N+2}^{\infty} \sum_{j=n}^{N} \frac{1}{q(j)} f(k, \mathbf{x}(k)) .
\end{aligned}
$$

It is clear now that, again by (H3) and (H5), we can take limits as $N \rightarrow \infty$ and, doing so, see that $\mathbf{x}$ is a solution of equation (2).

Let us now state and prove the main result of this paper.

Theorem 3 Under conditions (H1)-(H6), equation (E) has a solution in $\ell_{\infty}(X)$.

Proof First we get rid of the case in which, for one reason or another, the sum in the right hand side of equation (E) is always missing (equal to 0 for all $n$ ),

$$
\mathbf{x}(n)=g(\mathbf{x}) .
$$

An $\ell_{\infty}(X)$ solution to this equation must be a constant sequence $\mathbf{c}^{x}$, given by $\mathbf{c}^{x}(n)=x$, $n \in \mathbb{N}$, satisfying $g\left(\mathbf{c}^{x}\right)=x$. To this aim, define $\tilde{g}: X \rightarrow X$ as $\tilde{g}(x)=g\left(\mathbf{c}^{x}\right)$. By $(\mathrm{H} 1), \tilde{g}$ is clearly 
compact and bounded, showing that $\overline{c o}(\tilde{g}(X))$ is a $\tilde{g}$-invariant nonempty, bounded, closed, and convex set. So by Schauder's fixed point theorem, $\tilde{g}$ has a fixed point $x_{0} \in X$ and, consequently, the corresponding constant sequence $\mathbf{c}^{x_{0}}$ is a solution of our equation.

From now on, we assume that the sum on the right hand side of equation (E) is not always 0 . Define $\mathbf{T}: \ell_{\infty}(X) \rightarrow \ell_{\infty}(X)$ as $\mathbf{T}=\mathbf{G}+\mathbf{S}$ where $\mathbf{G}(\mathbf{x})=\mathbf{c}^{g(\mathbf{x})}$, and each component of $\mathbf{S}=\left(S_{0}, S_{1}, \ldots\right)$ is $S_{n}(\mathbf{x})=-\sum_{k=n+1}^{\infty} P(n, k) f(k, \mathbf{x}(k))$. Observe that $\mathbf{G}$ is well defined, and so is each $S_{n}$ because conditions (H2), (H3), and (H5) tell us that the series defining $S_{n}$ converges absolutely for each $\mathbf{x} \in \ell_{\infty}(X)$, and does it uniformly on each bounded set of $\ell_{\infty}(X)$. Clearly, by (HH1), $\mathbf{G}$ is compact and bounded.

The plan for the rest of the proof consists in showing that $\mathbf{S}$ is $\alpha_{\infty}$-condensing. This will be achieved using the first part of Theorem 1. From here, it will be clear that $\mathbf{T}=$ $\mathbf{G}+\mathbf{S}$ is $\alpha_{\infty}$-condensing. Finally, we shall show that $\mathbf{T}$ satisfies a Leray-Schauder boundary condition (LS) on some closed ball, concluding that $\mathbf{T}$ has a fixed point, which necessarily is a solution to equation (E).

We initiate our route map showing that $\mathbf{S}=\left(S_{0}, S_{1}, \ldots\right)$ satisfies the conditions in Theorem 1. We start checking that $\mathbf{S}$ maps bounded sets into bounded sets. If $R>0$, and $\mathbf{x} \in \ell_{\infty}(X),\|\mathbf{x}\|_{\infty} \leq R$, then conditions (H2), (H3), (H5), and the fact that $\varphi$ is nondecreasing, by (H4), give, for $n \in \mathbb{N}$,

$$
\left\|S_{n}(\mathbf{x})\right\|_{X}=\left\|-\sum_{k=n+1}^{\infty} P(n, k) f(k, \mathbf{x}(k))\right\|_{X} \leq \varphi(R) \sum_{k=n+1}^{\infty} p(k) a(k),
$$

concluding that $\mathbf{S}\left(\bar{B}_{\ell_{\infty}(X)}(\mathbf{0}, R)\right) \subset \bar{B}_{\ell_{\infty}(X)}\left(\mathbf{0}, \varphi(R) \sum_{n} p(n) a(n)\right)$.

The diameter condition on a bounded set, say contained in a ball of radius $R$, also follows from the above argument, for the right hand side in (4) goes to zero as $n \rightarrow \infty$.

Next, for fixed $n \in \mathbb{N}$, we show the continuity of $S_{n}$ at a fixed $\mathbf{x}_{0} \in \ell_{\infty}(X)$. Let $\varepsilon>0$ be given. Take $R_{0} \geq\left\|\mathbf{x}_{0}\right\|_{\infty}+1$. Since $\sum_{k} p(k) a(k)<\infty$, there exists $k_{0} \geq n+1$ such that $2 \varphi\left(R_{0}\right) \sum_{k=k_{0}+1}^{\infty} p(k) a(k)<\frac{\varepsilon}{2}$. Now there exists $\delta>0$ such that for each $k \in\left\{n+1, \ldots, k_{0}\right\}$, as the function $f(k, \cdot)$ is continuous at $\mathbf{x}_{0}(k)$,

$$
p(k)\left\|f(k, x)-f\left(k, \mathbf{x}_{0}(k)\right)\right\|_{X}<\frac{\varepsilon}{2 k_{0}}, \quad \text { whenever } x \in X \text {, and }\left\|x-\mathbf{x}_{0}(k)\right\|_{X}<\delta .
$$

All this implies that if $\mathbf{x} \in \ell_{\infty}(X)$ is such that $\|\mathbf{x}\|_{\infty}<R_{0}$, and $\left\|\mathbf{x}-\mathbf{x}_{0}\right\|_{\infty}<\delta$, then

$$
\begin{aligned}
\left\|S_{n} \mathbf{x}-S_{n} \mathbf{x}_{0}\right\|_{X} & \leq \sum_{k=n+1}^{\infty} P(n, k)\left\|f(k, \mathbf{x}(k))-f\left(k, \mathbf{x}_{0}(k)\right)\right\|_{X} \\
& \leq \sum_{k=n+1}^{k_{0}} p(k)\left\|f(k, \mathbf{x}(k))-f\left(k, \mathbf{x}_{0}(k)\right)\right\|_{X}+2 \varphi\left(R_{0}\right) \sum_{k=k_{0}+1}^{\infty} p(k) a(k) \\
& <\sum_{k=n+1}^{k_{0}} \frac{\varepsilon}{2 k_{0}}+\frac{\varepsilon}{2} \leq \varepsilon,
\end{aligned}
$$

proving the continuity of $S_{n}$ at $\mathbf{x}_{0}$.

Now, for fixed $n \in \mathbb{N}$, we prove the condensing property of $S_{n}$. Let $\mathbf{A}$ be a bounded set in $\ell_{\infty}(X)$ with $\alpha_{\infty}(\mathbf{A})>0$. Then $S_{n}(\mathbf{A})$ is bounded in $X$. Write $A_{k}$ for the $k$ th projection 
of $\mathbf{A}$ onto $X$, and notice that $\alpha_{X}\left(A_{k}\right) \leq \alpha_{\infty}(\mathbf{A})$, for if $\varepsilon>0$ is such that there exists a finite $\varepsilon$-cover of $\mathbf{A}$, then the $k$ th projections of this cover provide a finite $\varepsilon$-cover (in $X$ this time) of $A_{k}$. Also notice that

$$
S_{n}(\mathbf{A})=\left\{-\sum_{k=n+1}^{\infty} P(n, k) f(k, \mathbf{x}(k)): \mathbf{x} \in \mathbf{A}\right\} \subseteq-\sum_{k=n+1}^{\infty} P(n, k) f\left(k, A_{k}\right),
$$

and that this last set is bounded; so, using (H2) and the algebraic properties of the Kuratowski measure of noncompactness, we have for any $N \in \mathbb{N}, N \geq n+1$,

$$
\begin{aligned}
\alpha_{X}\left(S_{n}(\mathbf{A})\right) & \leq \sum_{k=n+1}^{N} P(n, k) \alpha_{X}\left(f\left(k, A_{k}\right)\right)+\alpha_{X}\left(\sum_{k=N+1}^{\infty} P(n, k) f\left(k, A_{k}\right)\right) \\
& \leq \sum_{k=n+1}^{\infty} p(k) \alpha_{X}\left(f\left(k, A_{k}\right)\right)+\operatorname{diam}\left(\sum_{k=N+1}^{\infty} P(n, k) f\left(k, A_{k}\right)\right) .
\end{aligned}
$$

Observe that as $N \rightarrow \infty$, the summand on the right goes to 0 . So, by condition (H6) and the fact that $\alpha_{X}\left(A_{k}\right) \leq \alpha_{\infty}(\mathbf{A}), k \in \mathbb{N}$, obtain

$$
\begin{aligned}
\alpha_{X}\left(S_{n}(\mathbf{A})\right) & \leq \sum_{k=n+1}^{\infty} p(k) \alpha_{X}\left(f\left(k, A_{k}\right)\right) \leq \sum_{k=n+1}^{\infty} p(k) L_{k} \alpha_{X}\left(A_{k}\right) \\
& \leq \sum_{k=n+1}^{\infty} p(k) L_{k} \alpha_{\infty}(\mathbf{A})<\alpha_{\infty}(\mathbf{A}) .
\end{aligned}
$$

According to our route map, to finish the proof, we just need to show that $\mathbf{T}$ satisfies the Leray-Schauder boundary condition on $\bar{B}_{\ell_{\infty}(X)}(0, R)$ for some $R>0$. To find what conditions must satisfy such $R>0$, assume that there exist $\lambda>1$ and $\mathbf{y} \in \ell_{\infty}(X)$, with $\|\mathbf{y}\|_{\infty}=R$ and $\mathbf{T y}=\lambda \mathbf{y}$.

By (H1), $g$ is bounded, so there is $M_{g}>0$ such that $\|g(\mathbf{x})\|_{X} \leq M_{g}$ for all $\mathbf{x} \in \ell_{\infty}(X)$. Now, for $n \in \mathbb{N}$, using the hypotheses at our disposal,

$$
\begin{aligned}
\|\mathbf{y}(n)\|_{X} & =\frac{1}{\lambda}\|\mathbf{T y}(n)\|_{X} \leq \frac{1}{\lambda}\|\mathbf{G y}(n)\|_{X}+\frac{1}{\lambda}\|\mathbf{S} \mathbf{y}(n)\|_{X} \\
& \leq \frac{1}{\lambda}\|g(\mathbf{y})\|_{X}+\frac{1}{\lambda}\left\|-\sum_{k=n+1}^{\infty} P(n, k) f(k, \mathbf{y}(k))\right\|_{X} \\
& <M_{g}+\frac{1}{\lambda} \sum_{k=n+1}^{\infty} p(k)\|f(k, \mathbf{y}(k))\|_{X} \\
& \leq M_{g}+\frac{1}{\lambda} \sum_{k=n+1}^{\infty} p(k) a(k) \varphi\left(\|\mathbf{y}(k)\|_{X}\right) .
\end{aligned}
$$

The technique we are about to use now consists in applying an infinite discrete analog of the well-known Gronwall-Bellman-Bihari inequality, which has extensively been studied by Pachpatte (see, for instance, [13]), especially for finite difference equations. We just show the method for the sake of completeness. 
Let $z: \mathbb{N} \rightarrow \mathbb{R}$ be defined, for each $n$, as the rightmost member of (5). Observe first that $z(n)>\|\mathbf{y}(n)\|_{X} \geq 0$, for all $n$, and that $z(n) \rightarrow M_{g}$ as $n \rightarrow \infty$ because $\sum_{k} p(k) a(k)<\infty$ and $\varphi\left(\|\mathbf{y}(k)\|_{X}\right) \leq \varphi\left(\|y\|_{\infty}\right)$. Also, $z$ is a nonincreasing sequence, for

$$
z(n)-z(n+1)=\frac{1}{\lambda} p(n+1) a(n+1) \varphi\left(\|\mathbf{y}(n+1)\|_{X}\right) \geq 0 .
$$

Since $\varphi$ is nondecreasing, $\varphi\left(\|\mathbf{y}(n)\|_{X}\right) \leq \varphi(z(n))$ for all $n$, and since $\varphi$ is positive on positive numbers, dividing by $\varphi(z(n+1))>0$ in (6), and using again the nondecreasing property of $\varphi$, we obtain

$$
\frac{1}{\lambda} p(n+1) a(n+1) \geq \frac{z(n)-z(n+1)}{\varphi(z(n+1))} \geq \int_{z(n+1)}^{z(n)} \frac{1}{\varphi(s)} d s .
$$

Summing up to $\infty$ in the above inequality, we get, putting $\Psi(t)=\int_{1}^{t} \frac{1}{\varphi(s)} d s$,

$$
\frac{1}{\lambda} \sum_{k=n+1}^{\infty} p(k) a(k) \geq \sum_{k=n}^{\infty} \int_{z(k+1)}^{z(k)} \frac{1}{\varphi(s)} d s=\int_{M_{g}}^{z(n)} \frac{1}{\varphi(s)} d s=\Psi(z(n))-\Psi\left(M_{g}\right) .
$$

Observe that $\Psi$ is a strictly increasing function from $[1, \infty)$ onto $[0, \infty)$, because $\int_{1}^{\infty} \frac{1}{\varphi(s)} d s=\infty$, so $\Psi^{-1}$ is a well-defined strictly increasing function from $[0, \infty)$ onto $[1, \infty)$. Therefore, for $n \in \mathbb{N}$,

$$
\begin{aligned}
\|\mathbf{y}(n)\|_{X} & \leq z(n) \leq \Psi^{-1}\left(\Psi\left(M_{g}\right)+\frac{1}{\lambda} \sum_{k=n+1}^{\infty} p(k) a(k)\right) \\
& \leq \Psi^{-1}\left(\Psi\left(M_{g}\right)+\sum_{k} p(k) a(k)\right) .
\end{aligned}
$$

So it just suffices to take at the beginning $R>\Psi^{-1}\left(\Psi\left(M_{g}\right)+\sum_{k} p(k) a(k)\right)$ so as to arrive at the contradiction of assuming also $\|\mathbf{y}\|_{\infty}=R$. This finishes the proof of the theorem.

As an easy consequence of this theorem, we present now a result about existence of solutions for the second order difference problem (1).

Corollary 1 With $P(n, k)=\sum_{j=n}^{k-1} \frac{1}{q(j)}$, for $k \geq n+1$, where $q$ is a sequence of positive numbers, under conditions (H1)-(H6), the second order difference problem (1) has a solution $\mathbf{x} \in \ell_{\infty}(X)$.

For the proof, first find a solution of the corresponding difference equation (E) or, for the case, of equation (2). Then this solution is also a solution of (1).

Remark 2 As we mentioned before, Corollary 1 includes the well-known problem on the existence of asymptotically constant solutions, just taking $g(\mathbf{x})=C$, with $C$ a constant. Obviously, the situation covered by our result is more general, and this can be seen considering a bounded, continuous, and nonconstant $g: \ell_{\infty}(X) \rightarrow X$. To obtain an explicit example of this type, take a continuous and bounded map $g_{0}: \mathbb{R} \rightarrow \mathbb{R}$ and $u \in X$, and define $g: \ell_{\infty}(X) \rightarrow X$ as $g(\mathbf{x})=g_{0}\left(\|\mathbf{x}\|_{\infty}\right) u$, or $g(\mathbf{x})=g_{0}\left(\|\mathbf{x}(1)\|_{X}\right) u$. Then it is easily checked that $g$ is bounded and compact. 
Competing interests

The authors declare that they have no competing interests.

\section{Authors' contributions}

All authors contributed equally to the writing of this paper, and all authors read and approved the final manuscript.

\section{Acknowledgements}

Research was partially supported by the Spanish (Grant MTM2014-52865-P) and regional Andalusian (Grant FQM210) Governments.

Received: 4 May 2016 Accepted: 4 September 2016 Published online: 13 September 2016

\section{References}

1. Gil', M: Difference Equations in Normed Spaces. Elsevier, Amsterdam (2007)

2. González, C, Jiménez-Melado, A: Set-contractive mappings and difference equations in Banach spaces. Comput. Math. Appl. 45, 1235-1243 (2003)

3. Drozdowicz, A, Popenda, J: Asymptotic behavior of the solutions of the second order difference equation. Proc. Am. Math. Soc. 99, 135-140 (1987)

4. McKee, S, Popenda, J: On the existence of asymptotically constant solutions of a system linear difference equations. Fasc. Math. 28, 109-117 (1998)

5. Bezubik, A, Migda, M, Nockowska-Rosiak, M, Schmeidel, E: Trichotomy of nonoscillatory solutions to second-order neutral difference equations with quasi-difference. Adv. Differ. Equ. 2015, 192 (2015)

6. Popenda, J, Schmeidel, E: On the asymptotic behaviour of nonhomogeneous linear difference equations. Indian J. Pure Appl. Math. 28, 319-327 (1997)

7. Reynolds, DW: On asymptotic constancy for linear discrete summation equations. Comput. Math. Appl. 64, 2335-2344 (2012)

8. Schmeidel, E: An application of measures of noncompactness in investigation of boundedness of solutions of second order neutral difference equations. Adv. Differ. Equ. 2013, 91 (2013)

9. Ved', YA, Golovina, VG: Criterion for the existence of asymptotically constant solutions of systems of second-order difference equations. In: Imanaliev, MI (ed.) Studies in Integro-Differential Equations, vol. 16. "Ilim”, Frunze (1983) (in Russian)

10. Sadovskiï, BN: On a fixed point principle. Funkc. Anal. Prilozh. 1, 74-76 (1967)

11. Agarwal, RP, Meehan, M, O'Regan, D: Fixed Point Theory and Applications. Cambridge University Press, Cambridge (2001)

12. Ayerbe Toledano, JM, Domínguez Benavides, T, López Acedo, G: Measures of Noncompactness in Metric Fixed Point Theory. Birkhäuser, Basel (1997)

13. Pachpatte, BG: Inequalities for Finite Difference Equations. Dekker, New York (2002)

\section{Submit your manuscript to a SpringerOpen ${ }^{\circ}$ journal and benefit from:}

- Convenient online submission

Rigorous peer review

- Immediate publication on acceptance

- Open access: articles freely available online

- High visibility within the field

- Retaining the copyright to your article 